\title{
Ueber Cysticercus cellulosae cerebri et musculorum, mit besonderer Berücksichtigung der den Parasiten einschliessenden Kapselwand.
}

\author{
Von \\ L. JACOBSOHN, \\ Borlin. \\ (Hierzu Tafel III-VIII.)
}

Vor mehreren Jahren liatte ich durch die Freundlichkeit eines meiner Schüler, Herrn Dr. Taniguchi aus Japan, Gelegenheit, Priiparate von Distomum cerebri zu sehen, die er in meinem Laboratorium ungefertigt hatte. Die Eigenschaften der Kapselwand dieses in Japun häufig beim Menschen vorkommenden Parasiten erweckten den Anschein, als ob es sich bei diesen Wandbestandteilen nicht ausschliesslich, der üblichen Annahme gemäss, um reines Bindegewebe handelt, sondern als ob diese Ka $K_{\text {pel- }}$ wand eine durch Entründung stark veränderte Gefässwand sei. Herr Dr. Taniguchi hat alsdann auch in der über diesen Gegen. stand handelnden Arbeit') den Bau dieser Kapselwand nälier beschrieben und die Umstïnde erliutert, welche zu der erwilinten Vermutung geführt haben. Indessen weiter als bis zu dieser Vermutung lionnten die Präparate niclıt führen, ein vollgültiger Beweis für diese Anschuuung war nicht zu erbringen.

Es war mir deshalb von grossem Interesse, als ich durch die grosse Liebenswürdigkeit meines Freundes Prof. L. Minor aus Moskau Gehirn- und Mluskelstücke einer an Cysticerkose verstorbenen Patientin erhielt. Die grosse Zahl der in diesen Stücken vorhandenen Cysten und die relative Kleinlieit der meisten derselben liessen von vornherein vermuten, dass man bei mikrosliopischer Bearbeitung des Materials der Entscheidung der aufgeworfenen Frage vielleicht näher kommen dürfte.

Der Krankheitsfall selbst ist klinisch von Herrn Dr. Preobraschensliy aus Moskau beobachtet und im Korsakoffschen Journal für Psychiatrie und Neurologie 1904 publiziert worden. In dieser Arbeit finden sich auch Angaben über den makroskopischen Selitionsbefund nebst wwei Abbildungen, welche die ausserordentliche Cysticesteninvasion im Gehirn und in Herzmuskel veranschaulichen ${ }^{2}$ ). Ferner sind in der Arbeit auch An-

"l'aniguchi, Ein Full von Distomua-Erlorankung des Gehirus etc. Arch. f. P.ych. B.I 38 . H. 1 . biliungen.

${ }^{2}$ ) Fig. 1, Taf. III, ist die Reproduktion einer dieser genannten $\Delta b$ - 
gaben über den mikroskopischen Befund gemacht worden, welche aber diejenigen Punkte, die hier niher erörtert werden sollen, niclst betreffen. Beiden Herren sage ich für die Ueberbringung des Materials und für die Ueberlassung desselben $\% u$ wissenschaftlicher Bearbeitung meinen besten Dank.

Nach einem, von Herrn Dr. Kron-Moskau erstatteten Referat über diesen Fill liandelt es sich um eine 28jithrige Biuluerin, die am 21. V. 1903 ins Hospital aufgenommen wurde. Anf:ung Jaunar 1903 litg sie $1^{1 / 2}$ Woclsen wegen "Febris typhoiden" in einem Krankenhaus. Eine Woche nach der Entlassung aus letzterem traten die Erscheinungen wieder auf und verinlassten Pat., abermals das Krankenhaus aufousuchen. Letrteres verliess sie nach einem Monat mit deutlichen Zeichen von Gedichtnisschwiiche. Seit dieser Zeit leidet sie an schnell vorübergehenden Ohnmachtsanwandlungen, an allgemeiner Schwache und psychischen Störungen. Diese Erscheinungen nahmen \%u, weshalb sie ins Hospital eintrat. Wiihrend des Krankenaufenthaltes wecliselten die Symptome bloss in ihrer Intensitit. Es reigten sich dort folgende Erscheinungen: Gediichtnisschwïche, Verwirrtheit, Jllusioneu, Halluzinationen und flüchtige Walhnvorstellungen. Hin und wieler traten Ohmmaclitsanfälle oder Konvulsionen auf. Patientin geht oder sit\%t bloss mit Unterstüt\%ung. Abgeselien ron einer 2-3 Tige dauernden Liilumung des linken N. Alducens, bestanden keine Paresen. Es bestand Staungspapille olme Sehschwäche. Die Patellarreflexe waren bald normal, bald schwer auslösbar. Im Dezember trat Verschlechterung des Allgemeinbefindens und $\mathrm{Zu}$ nalume der psychisclien Störungen ein. Gegen Ende der Krankheit entwickelte sich eine akute Tuberkulose. Exitus am 16. I. 1904.

A utopsie: Cysticercus cellulosae disseminatus im Cerebrum und in allen Muskeln (anch in Herz und Lunge), Tuberculosis acuta pulmonum. Auf der Oberflache der rechten Grosshirnbemisphäre waren etwa 250 Blasen zu sehen, auf der linken :a. 270; in der Hirnsubstan\% ungeheuer viele. Auf jedem Frontalsclınitt über 100 Blasen (Fig. 1, Taf. III). Die Gesamtzalıl der Blasen in beiden Hemisphären betriigt einige Tausend, es fanden sich auch Blasen in Plexus vasculosus, im Pedunculus cerebri, im Pons, in den Corpora quadrigemina und im Cerebellum. Dagegen waren in der Medulla oblongata und spinalis keine Blasen $z u$ finden. Auf der Herzolsertiche sassen etwa 120 Blasen, in der Herzmuskulatur melırere hunclert Blasen. In allen Muskeln, in den Kau-, Gesichts-, Zungen-, Hals-, Brust-, Rücken-, Bauch-, Diaphragmaund Extremitiitenmuskeln waren massenliaft Cysticerkenblasen. Eine Blase wurde unter der Magen- und eine anclere unter der Dünndarmschleimhaut gefunden. Im Unterhaut\%ellgewebe waren keine Blasen. Preobraschensky hebt die auffallende Tatsache bervor, dass trotz der kolossalen Invasion in Gelirn keine Lokalsymptome vorhanden waren.

Soweit das lieferat über die Arbeit von Preobraschensky. 
Von den mir übergebenen Stüclien aus Hirnrinde und von Muskeln fertigte ich Serienschnitte an, um den Bau der Cysticercusblase etwas niiher kennen zu lernen. Die meisten Schnitte wurden nach van Gieson gefärlot, einzelne auch mit Eosin-Alaun-Hämatoxylin, nach Weigerts Fïrbung der elastischen Fasern und nach der Weigert-Palschen Markscheidenfärbung.

Betraclitet man einen dieser Sclinitte mit dem blossen Auge oder mit der Lupe (Fiy.2, 'Tar. III), so hat er eine gewisse A elınlichkeit mit dem als Schweizer Käse (fromage de gruyère) bezeichneten Zustand. Es finden sich nämlich auf dem Schnitt zahlılreiche, meistens rundliche, aber aucl etwas anders gestaltete, grössere und kleinere, bald eng aneinander, bald etwas entfernt voneinander liegende Löcher. I) Unterschied zwischen dem eigentlichen Schweizerkäsezustand (oder dor ron Hartmann benannten cystischen Degeneration) und dem vorliegenden bestelit darin, dass hier die Löcher nicht leer sind, sondern als Inhalt den Durchschnitt des Cysticercus enthalten, und dass die Wand der Löcher nicht einfach Gehirnmasse ist, sondern dass sich eine wirliliche, bald dicke, bald diunne, bald glatte, bald gewundene Kapselwand findet.

Wenn viele Löcher, wie auf der Abbildung (Lig. 2, Talf. III) ersichtlich ist, entweder ganz leer sind oder ausser der Kapselwand nur eine dünne, hüllenartige Membran enthalten, so liegt das daran. dass einzelne Cysticerken schon in der Härtungsflüssigkeit aus den Löchern herausgefallen waren, und dass auch bei den vielen Manipulationen, denen später die Schnitte unterworfen wurden, einzelne Cysten jhren Inhalt einbüssten. In einem grossen Teil der Cysten war der Cysticercus nicht melır erhalten, sondern nur ein grösserer oder kleinerer Haufen von l'rümmerresten lag in der Kapsel drin (Fig. 3, Taf. III). Einzelne Cysten liegen so nube, dass sie nur noch durch eine Membran voneinander getrennt sind, mehrfach ist auch diese schon durchbrochen, so dass zwei oder mehrere Cysten ineinander übergehen und eine einzige, vielgestaltige Cyste aus ihnen entsteht (Fig. 2, 'Taf. III).

Die Kapselwand hat sich an vielen Cysten nicht überall gleichmässig ausgebildet. Neben geringeren Unterschieden an fast jeder Cyste findet man an einzolnen, dass die Kapselwand sich 7. B. an einer Stelle ungemein verdiclit hat, während an anderen das Gregenteil, eine Art Atrophie, eingetreten ist. Diese Atrophie kann so stark sein, dass dann überhaupt jede Spur von líapselwand fehlt. Wahrscheinlich durch postmortale Veriinderungen (auch durch die Einwirkung verschiedener, zum Härten etc. benutzter Rengentien) zeigt dann die benachbarte Hirusubstanz unregelmässige Einrisse, die der Cyste den sonst in sich abgeschlossenen Charakter nehmen (Fig. 2, Taf. III).

Sehen wir einstweilen von der Beschaffenheit der Kapselwand $a b$ und betrachten zunächst den Bau der in der Kapsel gelegenen Cysticercusblase. Da diese Blasen in vielen wohlerhaltenen Exemplaren in den uns zur Verfügung gestellten Stücken 
vorhanden waren, so konnte der Bau dieser Blase gut studiert werden.

Es wurden zum Zwecle einer genauen Einsichtnalıme Serienschnitte durch das Hirnstück, welches ich erlialten liatte (und welches nach seiner inneren IKonfiguration dem Corpus striatum nebst angrenzender äusserer Kapsel und Insel entsprach), gelegt.

Betrachtet man einen Schinit, welcher die Cysticercusblase etwa durch die Mitte ihrer Liingsachse getroffen laat (l'ig. 4, 'l'af. III), so hat man folgendes Bild: Der Kapselwand liegt eine lockere, schmale Hülle (a) ziemlich dicht an. Diese äussere Hülle geht an einem Pole (d) in eine innere Hülle (b) über. In dieser letrteren liegt der Hauptteil des Cysticercus (c) mit Kopf und Saugnäipfen. Die innere Hülle (b) liegt als eine kleine, eiförmige Blase in der grösseren äusseren Hülle, und da sie an einer Stclle (d) unter spitzem Winkel in letztere übergelit, so muss ilse Lage zur äusseren Hülle exzentrisch sein. Da die innere Hülle wiederum den Cysticercuskopf etc. umschliesst, so ist dessen Lage natürlich gleichfalls eine zur ganzen Blase exzentrische. Ein derartiges Bild bekommt man allerdings nur, wenn der Sclinitt, wie vorlier angegeben, durch die Längsachse des Cysticercus gegangen ist und dabei seine linstülpungsstelle (d) oder ileren Nachbarschaft getroffen hat. Ist das niclit der Fall, würde z. B. der Sclunitt den Cysticercus in der Verbindungslinie von b f, Fig. 4, Taf. III, getroffen haben, so würle einmal die innere Hülle niclıt exzentrisch in der ̈̈usseren, sondern ungefähr in der Mitte der letzteren liegen, und ferner wïrden beicle Hüllen vollkommen getrennt von einander arscheinen. Ein solches Bsld bietet die in Fig. 2 in der Mitte gelegene Cyste.

Wïluend der Raum zwischen der inneren (b) und äusseren Hülle (a), wenn der Schnitt die Cysticercusblase, sei es lïngs oder quer, jedoch durch die Mitte trifft, stets erlieblich gross ist, ist derselbe nur klein, wenn der Schnitt der Einstïlpungsstelle des Cysticercus (d) nahe liegt. Ein solches Bild stellt ungefiih. Pig. 5, Tal. IV, dar. Dieses Bild zeigt, dass die beilen Hüllen a und $b$ in der einen unteren Hälite $a^{\prime} b^{\prime}$ eine einrige Hülle uusmachen, wahrend sie im oberen leil durch einen schmilen Spalt getrennt sind. Liegt der Schnitt noch näher dem Pole zu, so ist der Cysticercus ringsherum nur von einer einrigen, aber breiteren Hülle umgeben. Diese Hülle hat einen der Kapselwand zugerichteten scharfen, dunkler gefïbten Rind (Fig. $5 a^{\prime}$ ) und einen lem Cysticercus zugewendeten, ebenso scharfen und ebenso dunklen liand (Fig. 5 $b^{\prime}$ ) und besteht in ilirem inneren, \% wschen den beiden Randstreifen gelegenen Abschnitt aus ganz lockerem Gewe';e. Dieses lockere Gewebe, welches also am Einstïlpungspol (Fig. $4 \mathrm{~d}$, Taf. III) resp. dessen Nachbarschaft die beiden Randstieifen zu einer gemeinsamen Hülle vereinigt, wird nun, wahrscheinlich durch Flüssigkeit, welche in ihre Maschenrïume tritt, bei Meiteren Wachstum des Cysticercus immer mehr gelockert. Schliesslich werlen die Maschenrïume durch immer grössere Ansammlung 
von seröser Flüssigkeit so gross, dass sich weite Cysternen bilden, die von einander nur durch feine, spinnwebeartige Septen getrennt sind (Fig. 4, Taf. III bei a). Auf einem Durchschnitt durch die Cysticercusblase erhält man dann den Eindruck, als wenn zwei Hüllen den Cysticercus umgeben, wihrend es, wie aus den Gesagten hervorgeht, in Wirlilichkeit nur eine ist '), in (leren inneres Maschenwerk Flüssigkeit getreten ist und dadurch ilıre Wäde auseinander getrieben hat. Diss dem so ist, erkennt man daraus, dass jede dieser beiden Hüllen an den sich \%ugekehrten Fliichen einen schmalen Saum von gleichartigem lockeren Gewebe enthält, dass hier und da eine schmale Brücke von lockerem Gewebe von der einen \%ur anderen Hülle hinüberfülıt, und dass in der Nailıe des Einstülpungspoles beide Säume zu einem gemeinsamen zusammen. fliessen (Fig. 4 d, T'af. 111, und Fig. $5 a^{\prime} b^{i}, T^{\prime} a f$. IV).

In diesen beiden, oder wenn man nach dem Auseinandergreset\%ten will, in dieser einen schlauchartigen und ringförmig gebogenen Hülle liegt nun der Hauptbestandteil des Cysticercus eingestülpt drin. Selır einfach wird dies fürs erste durch Fig.5 und liig.6, Tat. IV, veranschaulicht. Der Hauptbestandteil des Cy'sticercus ist hier der Kopf mit dem Hakenkranz, und den vier Saugnäpfen. Diese Gebilde, in Zentrum gelegen, sind von einer Srlieide von dichten, starken liasern ungeben (Fig. 5 f und 6f, Taf. IV), welche nuch aussen hin allmählich in lockeres Gewehe übergehen. Dieses lorkere Gewebe geht bei d (Fig. 5) in das gleichartige lockere Gewebe der den Cysticercus umfassenden gemeinsamen Hülle über, während der Uebergang auf der gegenüberliegenden Seite bei e, da lies die gemeinsame Hälle in eine äussere und innere gespalten ist, natürlich nur in die innere verfolgt werden kann. Dieses lockere Gewebe umricht aber auch ringsum den Bandwurmkopf (Fig. 6d) und schliesst wiederum, wie es bei den vorher beschriebenen Hüllen gewesen ist, mit einem schmalen, dunklen Rande (c) ab, und rieser liand geht lontinuierlich in den homologen von $b$ über. Vergegenwärtigen wir uns, dass dieser liand b an einer nnderen Stelle (Fig. 4 d, 'l'af. III) in den Rand a übergeht, und dass a und b die iusseren Flächen eines schlatuchartigen Gebildes sind, dessen Innenraum durch lockeres Gewebe erfüllt ist, so ergibt sich daraus, dass an einer hestimmten Stelle dieses schlauchartigen Gebildes, wo der I opf des spïteren Bandwurmes sich entwickelt, eine Einstülpung eingetreten sein muss (Fig. 4d, Taf. III) und dass in der daclurch entstandenen grubenartigen Aushöhlung der Kopf sich empor entwiclielt haben muss.

Hat nun der Schnitt die Cysticercusblase ungefähr in der Mitte, sei es in der Lïngs- oder Querrichtung, getroffen (Schnitte, wie sie durch die Fig. 4 veranschaulicht werden), so sieht man, dass der Hauptbestandteil des Cysticercus ausser dem Kopf (derselbe kann natürlich auch felılen, wenn er auf dem Schnitt nicht gerade witgetroffen ist) noch eigenartige, wie vielfach gewundene Drüsen-

1) Auch diese ist, wie wir spitter seben werlen, uur cine scheinbare Hülle. 
giinge aussehende Gebilde enthiilt. Betrachtet man nun die einzelnen Zotten dieser vermeintlichen Drüsengänge nälıer, so bestelıen sie aus einem dem Lumen des Ganges zugewanden scharfen, schmalen, dunkler gefärbten hande und einem sich anschliessenden lockeren Gewebe. Jer äussere, schmale Ranclsaum der zottenatigen Gebilde gleicht vollkommen in seiner Breite, seiner Farbe, seiner volliommen homogenen Beschaffenheit demjenigen Rande, welcher an den beiden, den Cysticercus einschliessenden Ilüllen zu beobachten ist, und ebenso ist auch das lockere Gewebe in seiner Art identisch demjenigen, welches an den lieiden oder an der einen gemeinsamen Hïlle zu sehen ist. Der Hauptunterschied in dem Bau dieser im Inneren des Cysticercus vorlandenen rottenartigen Gebilde und demjenigen der Hüllen bestelit larin, dass die Hüllen bis auf ihre Umsclılagstellen (lïg. $4 \mathrm{~d}$ ) eine riemlich glatte, gestreckte Oberfläche haben, wilhend die Zotten in Inneren vielfältig gewunden sind, und ferner darin, dass das lockere Gewebe in den Zotten niclit so stark gelockert ist wie in den Hüllen, weil es noch ron etwas derberen Faseriugen, die ron der derben Scheide (siehe rorher) ausgrehen und in die ein\%elnen Zotten hineineinstrahlen, fester \%usammengehalten wirl. Wälıend nun in sehr vielen Schnitten diese, im Inneren des Cysticercus gelegenen drūsenartigen Gänge von dem Ratume getrennt erscheinen, der zwischen innerer Hiille Fig. $4 \mathrm{~b}$ und dem Haupthestandteil des Cysticercus (Pig. 4 c) liegt, sielit man an anderen Schnitten, besonders an solchen, die in der Nähe der beiden Pole geführt sind, dass diese Gïnge in diesen erwähnten liaum einmünden (Fig. Td, lat. IV). Diese Schnitte im Verein mit solchen, welche durch die Einstülpungsstelle gelegt sind, geben zusimmen mit dem Umstande, dass die gewundenen, zottenartigen Gebilde im Inneren des Cysticercus in ihrem liau vollkommen demjenigen der Hüllen gleichen, den Schlüssel, wie die auf den einzelnen Schnitten etwas kompliziert aussehenden Bilder \%u deuten sind, d. h. welche Wandlungen die Cysticercusblase in ihrem weiteren Wachstum in ihrer äusseren Gestalt durchmacht, um sich in einem abgeschlossenen Raume möglichst ausbreiten zu können.

Die aus dem Ei der 'Taenia sich entwichelnde Onkosplaäre, welche auf dem Wege der Bluthahn ins Gehirn verschleppt wird, stellt ein lïngliches, schlauchartiges Gebilde dar. Dieses Gebilde bestelit nur aus einer ausseren glatten Haut und enthäl im Inneren ein nit Flüssigkeit erfülltes lockeres Gewebe. Dieses schlauchartige Gebilde hat ausserdem an einem Ende die Anlage für den späteren Kopf. Kopf- und Schwanzende des Schlauches krümmen sich so gegen einander, dass sie einen niclit vollkommen geschlossenen Ring hilden. Bei weiteren Wachstum wandert der Kopt in den Innenraum des Ringes hinein, den schlauchartigen Leib immer nach sich riehend und diesen Innenraum in hundertfiiltig verschiedenen Windungen durchmessend. Der liing bildet schliesslich eine gleiclumässige mantelartige Umhüllung der in seinem Innern gelegenen vielfachen Windungen. Indem der Ring, der also das 
caudale Ende des schlauchförmigen Parasiten darstellt, viel Flüssigkeit in seinem lockeren Gewebe anfnimmt, trennt sich seine äussere Lamelle mehr und mehr von seiner immeren. Zwischen beiden bildet sich ein weiter, blasenförmiger Raum, der den grössten Teil der Cysticercusblase ausmacht. Dieser Raum verengert sich nur an derjenigen Stelle, an welcher der Kiopf in den Innenraum des Ringes eingewandert ist (Einmindungsstelle Fig. 4 d, Tat. III). Durch diese Aufblähung des Ringes zu einer grossen Blase kommt es zuwege, dass nur seine innere Lamelle dem gewundenen Parasiten anliegt, Wilurend die iussere l,auselle durch einen weiten liaum von ihm getrennt ist. Diese Lamellen des Ringes scheinen später einem regressiven Pro\%ess anlieimoufallen, iusofern die beiden Schichten schmäler und lockerer werden, als diejenigen der im Inneren gelegenen gewundenen Einfaltungsgänge des Cysticercus. Nur cias muss min festhalten, dass diese Hüllen reiter nichts sind, als das Ende des Cysticerkenschlauches.

Ausser den genannten beiden Bestandteilen des Cysticercusschlauches (natürlich alıgesehen vom liopl), nämlich des ïusseren, schmalen, glatten, sich dunkel färbenden hindstreifens und der inneren Zoue von lockerem Gewebe, erwihnte ich schon, dass in dies lockere Gewebe sich noch Büschel von starken Fasern ergiessen, welche besonders die lioptregion mit einer starken Scheide umschliessen (Fig. $5 f^{\circ}$ und $6 \mathrm{f}$, l' $\mathrm{l}$ i. IV). Man findet ferner in clem lockeren Gewebe eigentümliche, sich mit slaunhämatoxylin stark violett färbende, kleine, ovale Körperchen liegen, die insgesamt das Bild ron kleinen, im Streu liegeuden Eiern gewähren. Diese kleiuen, eierartigen Gebilde trifft man auch vielfich an der Stelle, wo der freie liand der inneren llülle in den freien Rand des eingestïlpten Cysticercus umschlïgt (Fig. big, 'lanf. IV). Ja, bisweilen liegen sie in der blindsackartigen Einstülpung der Umschlagstelle selbst drin, so dass es den Anschein hat, als ob sie rom Cysticercus ausgestossen worden sind. Man trifft dann weiter grössere, rundliche, mit Al:uunhïmatoxylin sich gleichfalls stark fïlbende Gebilde sowohl in den Einfaltungskianälen des Cysticercus, als aucl in dem Rium $z w i s c h e n$ innerer Hülle und eingestülptem Cysticercus, als schliesslich zwischen äusserer Hülle und Kapselwand an. Die Gebilde haben einen konzentrischen Bau, und wenn sie zwischen iusserer Hülle und Kapselwand liegen, kerben sie erstere an der Stelle buchtartig ein (Fig. 8, Taf. IV). Wenn mehrere derartige Gebilde in gewissen Abstïnden zwischen ïusserer Hülle und Kapselwand liegen, so macht es den Lindruck, als ob erstere sich gleichsam an ilinen fest verankert hat. Es handelt sich bei diesen Gebilden wahrscheinlich um Ausscheidungsprodulte des Cysticercus. Welcher Natur aber dieselben sind, konnte nicht ermittelt werden. Hat die Kapselwand einen etwas rerklültet lamellösen Jau, so kann man sio auch einzeln zwisthen diesen Lamellen liegend funden. Ueber den Bandwurmkopf und die Sinugnïptchen habe ich vichts Besonderes \%u sagen. Die Figg. 6, 'laf. IV, und 9, Taf. V, geben das bekannte Bild dieses Kopfes wieder. Auch ver\%iclite 
ich hier auf eine noch genauere Beschreibung der einzelnen Schichten, die in den Hüllen und im Inneren des Cysticercus eivzelne Besonderheiten haben.

Der anatomische Bau des Cysticercus in der Muskulatur ist genau derselbe, wie im Gehirn (Fig. 10, Taf. V). Hervorzulieben wäre nur, dass der Zwischenraum zwischen der sog. äusseren und inneren Hülle des Cysticercus in der Muskulatur ein bei weitem grösserer ist, als im Gehirn. Die Ursache liegt wohl daran, dass das lockere Muskelgewebe der Ausdehnungsfähigkeit dieses Raumes viel geringeren Widerstand entgegensetzt, als die kompakte Hirnsubstanz.

Bevor jch die Beschreibung des anatomischen Baues des Cysticercus abschliesse, möchte ich noch einen Punkt etwas nihlıer erörtern, der nir des Interesses wert erscheint. Der Ujmstand, dass viele Cysticerken frisch erlıalten waren, im Verein mit demjenigen, dass Serienschnitte angelegt waren, brachte Aufschluss darüber, in welcher Art die Ernährung des Parasiten in dem Virte. in dem er sich angesessen, yor sich geht.

Sieht man die Serie durch, so begegnet man einzelnen Prïparaten, in welchen die aussere Hülle entweder an der Einbuchitungsstelle oder in deren Nachbarschalt (zuweilen kann es auch in einiger Entfernung von dieser Stelle sein) einen regelmāssig und vielfach eingekerbten Rand zeigt. Kleine hügelartige Erhabenheiten wechseln gleichmässig nit ebenso leicliten Einbuclitungen ab. Diesen leichten Einluuclitungen der äusseren Hülle des Cysticercus entsprechen kleine legelfürmige Hügcl, welche sich von der gegenüberliegenden Wand der Kapsol erheben und sich in die Einbuchtungen der iusseren Hülle hineinlegen (Fig. 11, Tat. V). Münlet gerade dic Einfultungsöffnung des Cysticercus in diese Ansaugungsstelle les Cysticercus, so legt sich cin grösserer Hügel der Kapsel \%ungenartig in diese Einfaltungsöffuung hinein (Figg. 11 u. 12, 'lat. V). Die Kapselwand an dieser Ansaugungsstelle des Cysticercus zeigt gewöbnlich einen locker lamellüsen Bau. Zwischen den ein \%elnen Lamellen liegen entweder ganz feine Blutgefaisse, welche strotzend mit Blut gefüllt sind, oder aher die Blutliörperchen liegen gedrängt in den Maschen zwischen den Lamellen. Ausserdem liegen zwischen diesen Lamellen auch grosse rundliche Elemente, welche cinen ganz. kleinen Kern enthalten, sonst aber von fist homogener Beschaffen. heit sind. Diese Elemente, d. h. massenliaft Blutkörperchen, $z$ wischen welchen die grossen Elemente eingestreut liegen, erfüllen nun auch die buchtenartigen grösseren und lileineren läiume zwischen der äussereu Hülle? des Cysticercus und der Kapselwand, so dass man den Eindruck gewiunt, als ob an dieser Ansilugungsstelle einmal das Nïhrmaterial in den Cysticercus übergeführt, gleichreitig aber auch die Absonderungsstofie aus ihm herausgescluafft werden. Der ganze Bau dieser Ansaugungsstelle erinnert etwas an das Chorion der Siugetiere. 
Ich habe den Bau des eingefalteten Cysticercus hier etwas näler beschrieben, obwohl ich annelıme, dass dies von seiten der Zoologen schon genugsam geschehen ist, weil der Mediziner wohl selten Gelegenlieit hat, frische, wohlerhaltene Exemplare dieses Parasiten zu untersuclien.

Indessen, das Hauptinteresse dieser Arbeit ist nicht dem Bau des Cysticercus zugrewandt gewesen, sondern der Kapsel, welche ihn umschliesst. Dieser Gegenstand hat insofern ein allgemeineres Interesse, als die Kapselwand wie hier, so auch bei manchen anderen nicht infelitiösen embolischen Prozessen sich wahrscheinlich aus den gleichen Elementen zusammensetzen wird. Diese Kapselwand scheint mir, um es gleich vorweg zu sagen, bei vielen Cysticerken nichts anderes als die verïnderte Gefaisswand zu sein, in welche hinein der Fremdliörper verschlagen wurde, und lie sich sehr verschieden verändert, je nachdem der Cysticercus nur leicht reizend, oder reizend und die Kapselwand erweiternd, oder schliesslich stark entzündungserregend wirkt.

Selien wir uns nun einmal die Kapselroand in diesem Falle von Cysticerkenembolie des Gehirns an.

Die Kapselwand hebt sich durch ihe dunkle Fiirbung stets ganz schauf von der iusseren Hülle des Cysticercus ub, so dass sie nit let\%terer gar vicht 7.u verwechseln ist. Es tritt dies besonders hervor, wenn die aussere Hülle mehr oder weniger sich gefaltet und von der Kinpselwand abgehoben hat (Fig. 5, 'Tiaf. IV); ebenso deutlich in Cysten, deren Inhalt in Trümmer zerfallen ist und in denen die Trümmer bald in grösserer Menge (nit Haken dabei) angehiuutt sind (Fig. 3, Taf. III) oder sich nur noch in Spuren der Kapselwand anhaftend vorfinden (Fig. 13). Aber auch in solchen Cysten, wo die ïussere Hülle des wohlerhaltenen Cysticercus der Kapselwand anliegt (Fig. 4, Taf. III, und noch mehr Fig. 14, 'Taf. VI), hebt sich letzotere durch ihren viel dunkleren Farbenton vor der ersteren scharf heruus, und ist auch zwischen beiden gewöhnlich an dieser oder jener Stelle ein feiner Spalt erkeunbar, der diese beiden Elemente von eimander trennt.

Die Kapselwand wird also nicht, wie von vielen Autoren angenonmen wurde, von Bestandteilen des Cysticercus gebildet').

Wenn aber nicht aus Elementen des Parasiten, woraus bildet sich die Kapselwand dann? Auf diese Frage geben die Präparate dieses Filles, wie ich glaube, eine zienlich deutliche Antwort.

Die Kapselwand ist entweder von ziemlich gleichmässiger, glatter Bescliaffenlieit und von mittlerer Dicke, nenn die Cysticercusblase firisch erhalten ist und ihr dicht anliegt (Fig. 4, Taf. III; Fig. 8, Iaf. IV; Fig. 14, Taf. VI) oder aber sie zeigt ein gefaltetes Ausselien und ist dann von wechselnder Dicke, wenn die äussere Hïlle des Cysticercus sich zusammengefaltet hat (Fig. う,

ग) Was hier rom Cysticercus gesagt wird, gilt wahrscheinlich auch für die anderen Parasilun. 
Taf. IV) oller weun der Cysticercus abgestorben und in Trümmer zerfallen jst. In den letzteren Fiallen hat sie bald ein kompaktes, schichtenartiges Gefüge (Fig. 13, Taf. VI। oder sie \%eigt einen etwas locker lamellüsen Bau (Fig. 3, Taif. III). Schliesslich findet man einzelne Cysticerken, die nur zum kleinsten 'Teil in einer Kapsel zu liegen scheinen, wihluend der grösste Teil in einem nur in der Gehirnsubstanz befindlichen Loche z.u stecken scheint. Sieht man sich den liand dieses Loches aber etwis näher an (Fig. 15 und Fig. 16, 'laf. VI), so findet man auf grössere oder kleinere Strecken Reste einer vorhanden gewesenen Wand, die als feine geschlingelte Lamelle sich erhalten hat.

Zur Fïrbung wurde, einzelne Präparate atusgenommen, durchgehend das van Giesonsche Farligemisch benutzt. Dieses Gemisch hat bei einfichster technischer Anwendung den unvergleichlichen Vorteil, die verschiedenen Gewebsarten durch einen anderen Farbenton her:tuszuheben und dadurch als solche kenntlich zu inachen.

Von Elementen, welche als Bausteine der Kapsel gedient hahen konnten, kamen im Gehirn in Betrachat: erstens die Neuroglia, zweitens die Grefässe, drittens das adventitielle Bindegewebe. Da die Fiapselwand der Muskelcysticerlien ungefälu den gleichen Bau aufwies, wie diojenige der Gehinncysticerken, so war eine Beteiligung der Neuroglia schon von vornherein ausgeschlossen, ausserdem fand sich nach dieser Richtung auch kein Anhaltspunkt.

Es konnten also als Bausteine der Kapsel nur die Gefässe resp. das adventitielle Bindegewebe in Betracht kommen. Hierbei war also \% entscheiden, ob das Material, aus welchem die Kapsel besteht, von der ganzen Gefässwand oder nur von einem bestimmten Gewebsteil derselbon, speziell ron dem adventitiellen Bindegewebe, gebildet wird.

Betrachtet man den Durchschnitt, Lüngs- oder Querschnitt nach von Gieson gefürbten Gefüssen, so kamn man an ilunen gewölınlich 3 Schichten wahrnehmen, die sich durch einen anderen Farbenton von einauder ablieben. Die lockere Adrentitia zeigt einen scharlachroten Farbenton mit einem starken Stich ins Piolette, die feste, je nach der Grösse des Gefiisses verschieden starke Mediat \%eigt einen gelblich orler gelblich-bräunlichen Ton und die bei kleineren Gefïssen aus einer scharfrandigen Lamelle mit Endothel\%ellen beset\%te Intima einen blassgelblich-rosafarbenen 'Ton.

Vergleicht man mit diesem Aussehen der Gefässwände dasjenige der Kapselwand, so findet man in einzelnen atuch eineu dreischichtigen Bau, wobei alle Schichten gewölulich durch starke Wucherung verbreitert sind (Fig. 17, Taf. YjJ). Zu innerst gegen das Lumen, zuweilen mit cinem scharfen liande abschneidend, eine verdickte und leicht gewundene Lamelle; auf diese folgt eine breite, bei schwiichere. Vergrösserung wie porös aussehende, gelblich-brïunliche Zone und auf liese eine noch breitere, balil 
einschichtige, bald mehrschichtige Zone von scharlachroter Fürbung. Diese letztere Zone ist entweder von ganz kompaktem Gefüge oder aber von lockerem $\mathrm{Bau}$, oder schliesslich teils von lockerem, teils von festem Gefüge, wobei die lockere gewölınlich die am meisten peripher gelegene Zone einnimmt und in das lockere Gewebe der Nachbarschaft übergeht. Am besten ausgeprägt fand ich diesen dreischichtigen Bau an einzelnen Kitpseln der Muskelcysticerken (z. B. Fig. 17). Dieser dreischichtige Bau fndet sich aber niemals im ganzen Umkreis der Kapsel, sondern immer nur an einem melir oder weniger grossen Abschnitt derselben. Häufig sieht man, wenn man den Umkreis der Kapsel verfolgt, dass sich die Kapselwand nach der entgegengesetzten Richtung 7.u (also auf dem Querschnitt nach dem anderen Pole 2u) verschmälert (Fig. 10, 'Taf. V). Die innerste Lamelle verschwindet ganz, ist wenigstens nicht deutlich $z u$ erkennen; die mittlere Schicht reduziert sich langsam zu einer schmalen, gelblich-bräunlichen Schicht, und auch die äussere Zone hat sich zu einer schmalen, festen Schicht gleichsan zusammengedrückt und geht in das intermuskuläre lockere Bindegewebe über. Diese beiden letzten Schichten können sich bis auf ganz schmale, aneinandergepresst liegende glattwandige Lamellen redurieren, wobei häufg noch die äussere über die innere herübergelagert ist, so dass die mittlere gelbe schwer oder an einzelnen Stellen überhaupt nicht $\%$ erkennen ist.

Im Vergleich mit dem Querschnitt von daneben im lockeren intermuskulären Bindegewebe liegenden Gefïssen geht man wohl nicht fehl, wenn man die innere, leicht gewundene Lamelle der sewucherten Kapselwand mit der Intima, die porös aussehende gelblich-briiunliche Schicht mit der Media und die äussere scharlachrote, bald kompakte, bald lockere Schicht mit der Adventitia identifiziert. Diese Schichten erfahren auf der einen Seite durch Wucherung eine sehr starke Verbreitung, wilhrend in anderen Stellen eine leichte Verschnilierung durch einfache Ausdehnung erfolgt. Diese verschmälerten Stellen sind es vorvehmlich, die zumal im Muskelgewebe zucrst beim Betrachten Zweifel erwecken, ob man es mit einem Gefïsslumen zu tun hat oder ob es sich einfach um Muskelfasern handelt mit angrenzendem Bindegewebe, zwischen welchtn der Cysticercus sich einfach hineingelegt und weiter entwickelt hat.

Die Kapselwind der Gehirncysticerken ist in einzelnen Exemplaren ähnlich gebaut wie bei den Bluskelcysticerken (Fig. 13, (Taf. VI) aber nicht in ihrem ganzen Umfange, sondern nur an einzelnen Stellen. In Fig. 13 \%eigt die Stelle a b c \%. B. einen wehrschichtigen Bau, wälırend die Stelle e einen lockerlamellösen darbietet, wogegen schliesslich die Stelle f erkennen lüsst, wie sich aus dem Pamellösen Bau durch dichteres Zusimmentreten der Lamellen ein mehrschichtiger Bau ausbildet. Die innere geluliche poröse Schicht bei c dūrfte der Media (im reinen $\mathrm{Zu}$ stund) entsprechen, wälırend die dicke, dunklere Schicht b einem 
Gemisch von Bestandteilen der Media und Adventitia entspricht, weshalb der Farbenton dieser Schicht auch ein Gemisch ron Scharlachrot und Gelbbraun darbietet. Diese feste Schicht geht dann nach aussen zu in das lockere perivaskuläre Bindegewebe a über. Der inneren Schicht liegen an einzelnen Stellen Blutreste und Trümmer des zerfallenen Cysticercus an (d).

Während nun einzelne Kapselwände, wie eben geschildert, keinen gleichnässigen Bau in ihrem ganzen Umkreis darbieten, ist dieser Bau bei anderen ein rollkommen gleichartiger, entweder schmal glattwandiger (Fig. 4, 8, 10a, 14, 17) oder durchgehends lamellöser. Letzterer Bau finde ́s sich am häufigsten bei geschrumpften oder in Trümmer zerfallenen (ysticerken (Fig. 3 und 20). Der glattwandige findet sich dagegen bei wohlerhaltenen Cysticerken, bei denen sich der Raum zwischen den beschriebenen beiden Hüllen stark erweitert hat. Die Kapselwand dieses glattwandigen Typus schliesst nach innen, nach dem Lumen zu, mit einem scharfen Rande ab (Fig. 8 und 14), an welchen sich der glatte Rand der iusseren Hülle des Cysticercus anlegt. Mitunter liegt die Hülle der Wand so dicht an, dass sie beide wie verklebt erscheinen, meistens aber ist $z$ wischen beiden doch ein feiner Spalt sichtbar. Die Wand solcher Kapsel bildet ein schmales, kompaktes, festes Gefüge, nur nach aussen zu lockert sie sich ein wenig auf. Betrachtet man sie bei stärkerer Vergrösserung, so zeigt sie in ihrer iusseren, nach der Hirnsubstanz \%ugerichteten Zone einen lamellösen Bau, während sie nach innen durch eine feste, schnurartige Membran abgeschlossen ist. Diese schnurartige innere Membran erkennt man besonders deutlich an einzelnen Stellen, wo sie sich zufällig etwas von der übrigen Kapselwand abgeboben hat (Fig. 8), und sie bleibt wahrscheinlich auch als letzter Rest zurück, wenn die Kapselwand atrophiert (Fig. 15 und 16). Die in der Nïhe solcher glattwandiger Kapseln gelegenen Gefïsse (Fig. 14c) zeigen in ihrem Bau und Farbenton eine solche Achnlichlieit mit der Kapselwand, (Fig. 14b) dass man schon durch diese Aehnlichkeit dahin gefülrt wird, sie für gleichartige Elemente aufzufassen. An einzelnen Stellen scheinen solche Gefässe sogar direkt in die Kapselwand hinein zu münden (Fig. 18, Taf. VII). Diese plattwandigen Kapseln zeigen, wie erwiihnt, nur in ihrer iusseren Zone einen schichtenartigen Bau aus einzelnen Lamellen. Let\%tere sind dünn und glattwandig. Zwischen den Lamellen sind viele Blutkörperchen reihenartig eingelagert. An diese Lamellen schliessen sich noch weiter nach aussen dünne, mit Blut vollgepfropfte Kapillaren an.

Neben diesen KKapseln findet man andere, deren Wand einen ganz gewundenen Verlauf zeigt. A uch hier zeigen einzelne eine ausserordentlich grosse Aehnlichkeit mit stark erweiterten Gefïssen. Man vergleiche \%. B. Fig. 13 mit Fig. 19, 'Taf. VII. Die Wand solcher Kanseln setzt sich aus einzelnen Lamellen zusammen, die entweder dicht aneinander liegen (Fig. 5) oder ganz locker in konzentrischer Art nebeneinander herlaufen (Figg. 3, 13, 20). Ob diese locker lamellüs gefügten Kapselwände dadurch entstehen, 
dass die einzelnen Schichten des ursprünglich fest gefügten' Gefísses bei der weiteren Verwandlung etwas auseinandergetrieben werden, wobei das adventitielle Gewebe stälker sich vermehrt, oder ob diese lamellös gebaute Kapsel dadarch entsteht, dass immer neue Gefässsprossen in der Peripherie des antinglich durch den Embolus erweiterten kleinen Gefässes sich anreihen, ist nicht sicher in allen Fïllen zu entscheiden. Für den ersteren Entstehungsmodus könnten z. B. Bilder, wie Fig. 19, sprechen, wo man sieht, dass ein einfaches, nur erweitertes Gefäss, abgeselien von den verschiedenen Dickendurchmessern, fnst genau der KionGguration einer Kapselwand entspricht (vergl. hier\%u Fig. 13); für den zweiten Entstehungsmodus sprechen Bilder, wie lig. 3 oder Fig. 20 sie darbieten.

Yon den Kapselwänden mit diesen lamellösen Bau ist noch 7. erwilhnen, dass man besonder's da, wo die Lamellen dicht aneinander liegen, zwischen ihnen quer getroffene Gefässlumina antrifft (Fig. 5), die bald leer, bald mit Blut gefüllt sind und die wahrscheinlich vasa vasorum repräsentieren.

Hervorgehoben muss noch weiter werden, dass man auf Querschnitten, die unmittelbar peripher vom Pol einer Kapselwanc. liegen, eine Art Strahlenkrone von vielen, mit Blut vollgestopften Gefässen findet (Fig. 21, 'Taf. VIII).

Hïufig liegt ein etwas stärkeres Gefäss im Zentrum, und von diesem breiten sich lileinere radienartig nach :tussen hin aus. Extravaskulïre Blutungen waren nicht zu beobachten.

Elastische Fasern waren sehr spärlich und nur an vereinzelten Stellen der Kapselwand nachweisbar. Eine Elastica interna war nicht zu beobachten. Bezüglich dieser Fasern verhielten sich auch einzelne stark erweiterte Gefässe dieses Falles sehr verschieden. An demjenigen, das z. B. in Fig. 15, T'af. VJ, abgebildet ist, waren elastische Fasern nur fragmentweise sichtbar, während an demjenigen, welches in Fig. 19 abgebildet ist, eine sehr starke Elastica besteht.

Immerhin war es bemerkenswert, dass ausser gelegentlich hier und da doch zu beobachtenden spärlichen Resten solcher Fasern in der Kapselwand letztere bei dieser Weigertschen Fărbung genau den diffus-violetten Farbenton zeigte, wie ihn die Media und Adventitia der Gefässe darbieten, während die äussere Hülle des Cysticercus bei dieser Fürbung ganz blass gefärbt blieb.

Nach alledem was die Präparate dieses Falles zeigen, unterliegt es für mich keinem $Z$ weifel, dass die Kapselwand, welche den Cysticercus einschliesst, nicht ausschliesslich bindegewebiger Natur ist, sondern dass sie in vielen Fällen eine verinderte Wand des Gefässes ist, in welches der Parasit durch den Blutstrom vertrieben wurde und in welchen er stecken geblieben ist. Dieses Gefüss ist in der Mehrzahl der Fälle ron kleinen Umfange, es scheint in einzelnen Fällen dem Arteriensystem, in anderen dem Venensystem anzugehören. Der Parnsit im embryo- 
nalen Zustancle wird in manchen Fïllen die. Kapillaren des Gehirns passieren (ebenso wie er die Lungenkapillaren passiert), und wird, vermöge der Verlangsamung des Blutstromes, in einer kleinen Vene stecken bleiben, und in dieser, falls er Subsistenzmittel findet, sich weiter entwickeln, falls nicht in diesem oder jenem Entwickelungsstadium zerfallen.

Dass die Kapselwand vielfach eine verinderte Gefüsswand ist, dafür sprechen 1. die äussere Achnlichkeit zwischen beiden, die in vielen Fällen geradezu verblüffend ist; 2. das Einmünden von verstopften Gefïssen in die Kapselwand, resp. der Umstand, dass sich am Pol der Kapsel regelmässig ein gröberes und viele feinere verstopfte Gefüsse finden; 3. der Bau der Kapselwand, der in einzelnen Fallen wie ein Gefïss, einen dreischichtigen Bau zeigt, der in vielen Fiallen einen zweischichtigen Bau zeigt, wobei diese Schichten bei der Fürbung nach van Gieson den gleichen Farbenton zeigen wie die beiden iiusseren Schichten (Media und Adventitia) der Gefässe; 4. das Vorhandensein, wenn auch spärlicher elastischer Fasern und auch bei dieser Fürbung der gleiche Farbenton, wie ihn Gefïsse bierbei aufweisen; j. das Zurückbleiben einer feinen, leicht gewundenen, schuurartigen Lamelle bei Atrophie der Gefüsswand und das Bestehen einer inneren, etwas stirkeren, einfachen l,amelle bei den glattwandigen Kapseln.

Die äussere Hülle des Cysticercus, welche nach der vorangegangenen Analyse im Verein mit der innaren Hülle nichts anderes ist, als das kaudale Ende der Cysticercusblase, nimmt an der Kapselbildung nicht teil. Die äussere Hïlle kann zuweilen der Kapsel so dicht anliegen, dass sie wie verlilebt mit ilır erscheint, aber meistens ist ein feiner Spalt zwischen beiden vorhanclen. Es kann allerdings nicht in Abrede gestellt werden, dass hier und da die Verklebung dieser beiden Wandteile eine stiirkere sein liann, so dass einzelne Stüclie auch bei spaiterer Verwandlung des Cysticercus an der Kapselwand haften bleiben und dass, wenn bei Zerfall des Cysticercus einzelne Bröckel an der Kapselwand haften geblieben sind, sie bei der Weiterbildung der Kapselwand gleichsam histologisch nut verarbeitet werden (Fig. 13). Das sind aber nicht regelmässige Bestandteile der Kiapselwand, sondern der Grundbau derselben besteht aus den Elementen des Gefïisses.

Es ist schliesslich noch z.u erwïhnen, dass, wenn die Kapselwand sich nicht weiter entwickelt, wenn sie im Gegenteil atrophiert und dann \%erreisst, der Cysticercus auch in einzelnen Fällen aus dem ursprünglichen Gefäisslumen herausgelien und etwas in die bemachbarte Gehirnsubstanz eindringen liann (Fig. 22, Taf. VIII). Dann bildet sich gewölınlich nur an einer I Iälfte eine neue Kapsel und *war aus neu entstandenen kleinen Gefïssen, deren Lamellen sich aneinanderlegen. Dieser \ustritt aus einem Gefässe geschieht walurscheinlich oft, wenn der Parasit in ein Gefätss an der Peripherie des Gehirns oder in eines in der Nachbarschaft der Ventrikel verschlagen worden ist. Aber auch hier, wo der Cysticercus aus 
der ursprünglichen Gefïsskapsel heraustritt, bildet er sich eine neue wiederum aus lileinen Giefïssen.

Es ist ferner nicht ausgesclilossen, dass die Wiand des Gefüisses, in welches der junge Cysticercus verschliagen wurde, nicht uur partiell, sondern in einzelnen Fïllen auch total \%ur Atrophie liomust, und dass dann die Kapselwand in ihrem gan\%en Umfange aus neugebildeten Gefüissprossen rusammengeset\%t ist. Aber um es besonders zu betonen, auch in diesen Fïllen setz.t sich die Kapselwand nicht aus einfachem Bindegewebe, sondern aus Gefüsssprossen zusammen, die sich lamellenartig aneinander reilien und bald locker geordnet, bald dichter gefügt sind.

Wenn in einem Falle, in welchem der Cysticercus aus einem Gefäss herausgetreten ist, lieine Blutung erfolgt ist, so liegt das daran, dass Blutungen doch nur da entstehen können, "o Bluttüssigkeit in Bewegung sich befindet; wenn wun ein Parasit ein kleines Gefüss verstopft und verödet hat und dimn dies Gefäss in seiner Wand zerfüllt und der Parasit heraustritt, so ist es ja natürlich; dass kein Blut mit heraustritt, weil diese Stelle aus dem Blutkreislauf schon längst ausgeschaltet ist. Dass auch in den anderen Fällen bei Verstopfung von Gefüssen keine nennenswerten Infarkte entstanden sind, liegt daran, dass im Gehirn, speziell in der Hirnrinde, ein so ausgiebiger Collaterallireislauf vorhanden ist, dass bei Verstopfung so kleiner Gefüsse ein Abschneiden gewisser, selbst kleiner, Hirnpartien vom Blutlauf niclit eintreten kann. Darin und in dem Mangel an stärkeren Ent\%ündungserscheinungen liegt auch der Grund, weshalb die Markfaserung der von mir untersuchten Hirngegend keine nennenswerte Atrophic zeigte.

Zum Schluss möchte ich noch ein pair Worte über die weiteren Veründeruugen sagen, denen die Kapselwand des Cysticercus (und wahrscheinlich auch anderer Gehirnparasiten) unterliegt. Frische und verhältnismässig junge Fälle ${ }^{1}$ ), wie der vorliegende, kommen ja seltener zur Beobachtung. Am häufigsten trifft man solche Fïlle, wo sich der Cysticercus in eine mit Flüssigkeit oder mit Trümmern mehr oder weniger erfüllte Cyste verwandelt bat, deren Natur festzustellen nur aus der Anwesenheit von Haken gelingt. In solchen alten Cysten sieht die Kapselwand gan\% anders aus.

Der Liebenswürdigkeit des Herrn Kollegen Heuneberg verdanke ich ein Präparat einer solchen alten Cysticercusblase. Eine Stelle aus dieser Cyste ist in Fig. 23, Taf. VIII, wiedergegeben. Aus dem Vergleich dieser Cyste mit denjenigen des hier beschriebenen Falles erkennt man sofort, dass die Lamelle a Fig. 23 identisch der äusseren Hülle des Cysticercus ist. Auch sie besteht hier. aus einem scharfen, äusseren, dunkler gefürbten Rande und einer dem Lumen zugekehrten inneren Zone, die aus lockerem Gewebe besteht. Diese Hülle hat sich auf der rechten Seite des Priparates

1) Nach der Krankengeschichte zu urteilen, dūrfte das Alter der antersuchten Cysticerken nicht ganz ein Jahr betragen. 
mit scharfem Rande abgelıoben, ebenso sieht man auf der linken Seite ihren scharfen Rand, dagegen liegt sie an der dritten, quergestellten Seite der nach aussen befindlichen Substanz dicht an, nur hin und wieder ist zwischen Hülle und Substanz auch hier ein kleiner Spalt bemerkbar. Der zwischen den eben genannten Abteilungen der Hülle liegende Raum, Fig. 23 d, entspricht dem Raum zwischen äusserer und innerer Hülle des frischen Cysticercus. Dieser Raum hat hier durch vielfache Filtungen und Verschiebungen der Cyste eine vielgestaltige Form angerommen und ist an einzelnen Stellen, an denen zwei Blätter der Hülle bei dieser Einfaltung aneinander gekommen sind (Fig. 23 $a^{\prime}$ ), vollkommen obliterirt. Es ist nicht unmöglich, dass an solchen Stellen die beiden aneinander tretenden Blätter der ïusseren Hülle mit ihrem lockeren Gewebe fest verkleben. Es ergibt sich demnach auch hier, dass nicht die äussere Hülle des Cysticercus, obwohl sie mit der anliegenden Sulstanz mehr oder weniger verkleben kann, die IKapsel, in welcher der ganze Cysticercus ruht, bildet, sondern dass die nach aussen von dieser Hülle gelegene Wand diese Kapselwand ausmacht. In dem Prïparat Fig. 23, ist also nicht a resp. a und b c die Kapsel des Cysticercus, sondern sie wird nur von b und c gebildet. Diese Kapselwand wird in grossen und ganzen von zwei breiten Schichten gebildet, einer inneren, helleren (b) und einer äusseren dunkleren (c). Letztere geht nach aussen langsam in die normale Hirnsubstanz über. Die innere helle Substanz besteht aus spindelförmigen Elementen mit ovalen langgestreckten Kernen, nach dem Lumen $z u$ schliesst sie mit einer schmalen, etwas dunkler gefürbten Randzone ab, in welcher sich etwas heterogene Elemente (evtl. Abfallstoffe der ïusseren Hülle) finden. Die breite äussere Zone besteht aus einer ausserordentlich dichten Ansammlung von rundlichen Elementen, die einer perivaskulïren Entzündungszone entspricht, nur dass sich hier die rundlichen Elemente zonenweise ungemein zusammengeballt haben, um sich allmählich nach aussen hin zu lockern und in das normale Gerrebe überzugehen.

I) $\mathbf{a}$ in dieser alten Cysticercusblase, Fig. 23, die Lamelle a der ïusseren Hülle des Cysticercus entspricht, so müssen dië beiden Zonen $b$ und $c$ die veränderte Kapselwand darstellen: Durch diese Verïnderung wird eine vorher lamellöse Wand in eine Art dicken Schutzwall umge wandelt. Das ist die üblich als Demarkationszone bezeichnete Wand. Wie im einzelnen nach und nach diese Veränderungen sich vollziehen, darüber geben die Präparate keinen vollkommenen Aufschluss. Nur aus einem Vergleich der Figg. 16, 19, 13, 20, 3, 23 kann man sich ungefähr eine Vorstellung von den sich vollziehenden Veränderungen machen.

Das Präparat, welches in Fig. 2t, Taf. VIII, abgebildet ist, stellt einen kleinen Ausschnitt einer durch Distomum hepaticum bedingten Gehirncyste dar. a in Fig. 24 ist der in Trümmer zerfallene und in der Cyste liegende Parasit, und b c sind die Schichten der ihn ungebenden Kapsel. Dass dies die Kapselwand ist, geht 
wohl unzweifelhaft aus einem Vergleich mit derjenigen in Fig. 23 hervor. Hier wie dort sind die Schichten die gleichen und setzen sich aus den gleichen Grundelementen zusammen.

Da nun die Kapselwand der alten Cysticerkenblase (Fig. 23) aus einer Gefässwand sich entwickelt hat, und da die Kapselwand des Distomum derjenigen des Cysticercus vollkommen gleicht, so ist die Schlussfolgerung berechtigt, dass auch die Kiapselwand des Distomum weiter niclits ais eine verinderte Gefiisswand darstellt. Mit der Wand einer Blutcyste hat die Kapselwand des Cysticercus gar keine Aehnlichkeit, mit der Wand eines Abszesses nur eine entfernte. Die let\%tere stellt, wenigstens in frischem Zustande, eine einfache, einheitliche, gleichmässige, sehr breite Entzündungszone dar, die wellenartig nach der Peripherie zu fortschreitet, wihrend in den zentralen Partien sie sich in Blut und Eitermasse auflöst. Ob die Wand ailterer Gehirnabszesse eine grössere Aehnlichkeit mit der Kapselwand des Cysticercus besitzt, darüber kann jch nichts Bestimmtes aussagen, da ich derartige Präparate nicht besitze.

Erklärung der Abbildungen nach Photogrammen auf den Tafeln III-VIII.

F1g. 1. Frontalschnitt durch beide Hinterhauptslappen, die enorme Cysticerken-Invasion illustrierend. Aus der Arbeit des Herrn Dr. Preobraschensky (Korsakoffsches Journ. f. Psych. u. Neurol., 1904).

Fig. 2. Cysticerken des Gehirns. Lupenvergrösserung eines Schnittes durch das Corpus striatum.

Fig. 3. Trümmer einer zerfallenen Cysticercusblase mit gewundener Kapselwand. Im Trümmerhaufen liegen cinige Haken (a) als letzte erkennbare Reste des Cysticercus.

Fig. 4. Cysticercusblase durch die Mitte ilirer Lāngsachse getroffen. a) Aeussere, b) innere Hülle, c) eingestülpter Cysticercus, d) Einstülpungsstelle.

Fig. 5. Cysticercusblase in der Nühe eines Poles durchschnitten. a) Aeussere, b) innere Hülle, c) eingestülpter - Cysticercus; $a^{\prime}$ und b die dunkeln, scharfen, freien Rïnder der gemeinsamen Hülle, d) und c) lockeres Gewebe, $f$ ) dichtes Fasergewebe.

Fig. 6. Kopfteil der Cysticercusblase von Fig. 5, vergrössert. Bezeichnungen wie in Fig. 5. g) Rundlich-eiförmige; im lockeren Geivebe liegende Elemente.

Fig. 7. Schnitt durch eine Cysticercusblase unweit eines Poles. d) Ausmündungsstelle des vieliach eingefalteten Cysticercuskörpers in den von den Hüllen a b umsclilossenen Ràum.

Fig. 8. Rundliches, anorphes Gebilde zwischen Kapselwand und äusserer Hülle des Cysticercus, letztere buchtartig einstülpend.

Fig. 9. Durchschnitt durch den Kopf des Cysticercus mit Hakenkranz (in der Mitte) und vier umliegenden Saugnapien.

Flg. 10. Cysticerken in der Muskulatur; bei b) Kapselwand von mehrschichtigem Bau, der sich nach dem anderen Pole der Kapsel zu verschmälert.

Fig. 11. Ansaugungsstelle des Cysticercus an die Kapselwand (b). Bildung der letzteren aus einzelnen Gefïsslamellen.

Fig. 12. Zungenartiger Hügel, der sich $z$ wischen Kapselwand (b) und einer:

Einstūlpungssstelle des Cysticercus (a) hineinlegt, und der vón Blutkörperchen und einzelnen grösseren Elementen erfüllt ist. 
136 Weinberger, Oober sogenannte "Doppelbildungen" am Gohirn,

Flg. 13. Kapselirand ciner zerfallenen Cysticercusblase. a) Lockeres, lamelJōses Gewebe, b) dichtes Gefüge aus Elementen der Media und Adrentitia, c) lockeres Gewebe, im Farbenton der Media äholich sehend. d) Zerfallstrümmer, e) lamellüser Bau der Cssten wand, f) Zone, an welcher die Lamellen sich zu einer festeren Wand zusammenschliessen.

Fig. 14. Giatte Kapsel rand ciner Cysticercusblase (b) mit daneben gelegenen Blutgefússen (c). a) Acussere Hülle der Cysticercusblase.

Fig. 15. Cysticercusblasc mit atrophischer Kapselwand (rechts). Ein ernicitertes Gefüss (links).

Fig. 16. Kapsclwaud einer zerfallenen Cysticorcusblase, bestehend aus der dūnoen, leicht geschlingelten, an einer Stelle zerrissenen Gefässirand.

Fig. 17. Segmente aus zwei nebeneinander liegenden Mluskelcssticerken nebst ibren Kapselwānden. a) Aeussere Hüllen der Cysticerken, b) Kapsel. wand, c) Miskulatur.

Flg. 18. Einnūndungsstelle cines Gefüsses in die Kapselwand einer Cysticorcusblase.

FIg. 19. Ein erweitertes Gefüss der Hirnrinde.

Fig. 20. Wellenartig gewundene Kapselwand cines zerfallenen Cysticercus: nach innen \%u eine dunklere Zone, in welcher dic Lamellen dicht gofügt sind, nach aussen zu locker gelagerte Lamellen, die sich gleichmâssig anligern; im Inneren, der welligen Kapselwand anliegend, Trümmerhaufen des zerfallenen C'jsticereus.

Fjg. 21. Verstopfte Gefüsse am Pol ciner Cysticercusblase.

F1g. 22. Ein kleines, gewundenes, zerrissenes Gefüss (rechts). Im Inneren desselben und in der Nachbarschaft der zerrissenen Stelle Erweichungstrūmmer. Links davon die Cysticercusblase.

Fig. 23. Querschnitt durch einen Teil einer älteren Cysticercusblase (nach oinem Prāparat des Herrn Prof. Hen neberg). a) Aeussere Hüllo des Cysticercus, b) und c) inncre und aussere Zone der Kapselwand der Cysticorcusblase, d) Innenraum der Blase. a' Ausbuchtungsstellen der Cyste, wo 2 wei Faltenblätter der àusseren Hülle dicht aneinander gelagert und sowobl miteinander als auch mit der Kapselwand verklebt sind.

F1g. 24. Kleiner Segmentabschnitt einer Gehirnçste von Distomum hepaticum (nacb einem Präparat des Herra Dr. Taniguchi). a) Zeríllene Masse dos Distomum, im Boblraum der Cyste gologen, b) innere, c) äussere Zone der Kapselwand, d) Distomumei. 